\title{
Prevalência de ansiedade e depressão em estudantes de medicina da região norte-nordeste de Santa Catarina
}

\author{
Prevalence of anxiety and depression in medicine students from the north-northeast \\ region of Santa Canatrina
}

Pravalencia de ansiedad y depresión en estudiante de medicina en la región norte-noreste de Santa Catarina

Eduardo Bach Pinheiro ${ }^{1 *}$, João Geraldo Eto Figueiredo', Washigton Luiz Gomes de Medeiros Junior $^{1}$, Januaria Ramos Pereira Wiese ${ }^{1}$.

\section{RESUMO}

Objetivo: Descrever o número de acadêmicos, ingressados em um curso de medicina na região Norte de Santa Catarina, com transtornos de Ansiedade e/ou Depressão. Assim como seus métodos individuais para confrontar tais transtornos. Métodos: Trata-se de estudo transversal descritivo observacional, conduzido com 232 acadêmicos do primeiro ao oitavo semestre do curso. Os usuários responderam a escala de HAD, questionário de auto-preenchimento usado na triagem de Depressão e Ansiedade. Também foram coletados dados a respeito de uso de medicamentos e substâncias, atividades físicas e acompanhamento com psicólogos/psiquiatras. Resultados: A amostra apresentou 76 (32,75\%) e 23 (9,9\%) acadêmicos classificados como provavelmente ansiosos e depressivos, respectivamente. Ao mesmo tempo que, 87 $(37,5 \%)$ e $53(22,8 \%)$ acadêmicos na faixa definida como possível ansiedade e possível depressão. Conclusão: Os níveis de ansiedade e depressão nos acadêmicos de medicina avaliados se encontram elevados em comparação à população geral, assim como o uso de medicamentos e drogas. O estudo também sugere um aumento na prevalência dos transtorno com o avançar no curso.

Palavras-chave: Ansiedade, Depressão, Acadêmicos de medicina, Transtornos de humor, Uso de substâncias.

\begin{abstract}
Objective: Describe the amount of medicine students, ingresses in a medicina course from the northern region of Santa Catarina - Brazil, with anxiety or depressive disorders. As well as their individual methods for confronting said disorders. Methods: This was a cross-sectional study, conducted with 232 students enrolled in the first through the eight semester. These students filled a survey containing the HAD, a self administered survey used in the triage of depression and anxiety. They also answered questions regarding the use of medication, recreational drugs, physical activity and psychological/psychiatric accompaniment. Results: The sample showed that $76(32,75 \%)$ and $23(9,9 \%)$ of the academics are classified as probably having anxiety and depression, respectively. Concomitantly, it presented $87(37,5 \%)$ and $53(22,8 \%)$ of the student body in the range classified as possibly anxious and depressive. Conclusión: The anxiety and depression levels were elevated amongst the academics analyzed, when compared to the general population, in addition to the use of medication and drugs. The study also suggests an increase in the prevalence of these disorders with the advance of the course.
\end{abstract}

Key words: Anxiety, Depression, Medicine students, Mood disorders, substance use.

\footnotetext{
1 Universidade da Região de Joinville (UNIVILLE), Joinville - SC. *E-mail: dubachpinheiro@gmail.com
} 


\section{RESUMEN}

Objetivo: Describir cuantos académicos matriculados en un curso de medicina de la región Norte de Santa Catarina - Brasil, que presentan transtornos de Ansiedad o Depresión. Así como saber quais métodos utilizan para afrentar estes transtornos. Métodos: Se realizó un estudio transversal descriptivo y observacional. La muestra estuvo integrada por 232 alumnos matriculados desde lo primer semestre hasta a el octavo semestre de La universidad. Los participantes responderam un cuestionario con la escala de HDA, que es normalmente utilizada en el cribado diagnostico de depresión y ansiedad. También recogieron atos sobre medicación, drogas, actividad física y seguimiento con profesionales médicos o psicoterapêuticos. Resultados: La muestra presentó $76(32,75 \%)$ e $23(9,9 \%)$ estudiantes considerados ansiosos o depresivos, respectivamente. En lo mismo tiempo que $87(37,5 \%)$ e $53(22,8 \%)$ estudiantes fueran definidos con posible ansiedad y posible depresión. Conclusión: Los níveos de ansiedad y depresión en los alumnos de medicina avaluados encuentran-se aumentados en comparación a la población general, así como La utilización de drogas y medicamentos. El estudio también sugiere una elevación de la prevalencia de los transtornos en el decorrer del curso.

Palabras clave: Ansiedad, Depresión, Académicos médicos, Trastornos del estado de ánimo, Uso de sustancias.

\section{INTRODUÇÃO}

O vestibular para o curso de medicina é um dos mais concorrido do país. Segundo dados disponibilizados pelo Sistema de Seleção Unificada (SISU) o curso de medicina possui a maior nota de corte, sendo necessário atingir pontuação média de 821,66 para se ter aprovação (MINISTÉRIO DA EDUCAÇÃO, 2020). Quando o objetivo é alcançado um sentimento de alívio acompanha os novos graduandos que tanto buscam o título de se tornarem médicos. Porém no decorrer do curso o estudante começa a perceber que a jornada que acompanha os seis anos de graduação é árdua e complexa devido a altíssima carga horária além de todas as dificuldades que as matérias trazem, como provas e trabalhos (BASSOL AM, et al., 2017).

As grandes demandas intelectuais e emocionais, somadas a uma exaustiva e intensa carga horária de trabalho, fazem do treinamento médico um estresse amplamente reconhecido (MACHADO SLM, et al., 2019). A maior carga acadêmica, longas horas de trabalho, um ambiente competitivo, conflito entre o trabalho e a vida pessoal, a falta de atividades recreativas, estar longe de casa e problemas financeiros podem ter um efeito adverso sobre a saúde mental dos alunos. Pesquisas indicam um aumento da prevalência de transtornos mentais e uma menor qualidade de vida em estudantes de medicina em comparação com a população em geral ou com alunos de outros cursos (RIBEIRO AGS, et al., 2016).

Estimativas mostram que cerca de $28 \%$ dos acadêmicos ingressados em cursos de Medicina apresentam sintomas depressivos, e que ainda 10,34\% apresentaram ideação suicida (PAULA JA, et al., 2015). Quanto à ansiedade, alguns estudos mostram que $34,3 \%$ dos médicos em formação apresentam sintomas falsopositivos de ansiedade e 19,7\% apresentam sintomas sugestivos de ansiedade, sendo este número cerca de duas vezes maior que população em geral (VASCONCELOS TC, et al., 2015).

De acordo com outro levantamento ainda, $57,38 \%$ dos estudantes apresentam algum escore para ansiedade e depressão. Dentre as dificuldades mais relatadas, encontravam-se: qualidade de vida cotidiana; relações com familiares; adaptação à mudança de cidade; falta de apoio institucional; dificuldade em procurar auxílio; vontade de trancar o curso; e o uso de psicotrópicos (CAMPOS JCL, et al., 2020).

Apesar da alta prevalência de depressão e ansiedade, a maioria dos alunos não busca por acompanhamento com psicólogo e/ou psiquiatra a decorrer da graduação. A relutância em demonstrar vulnerabilidade mesmo na vigência de dispositivos institucionais de atenção à saúde e maior vivência nesta área é apontada como causa de muitos discentes não procurarem esse tipo de suporte (ROBERTO A, et al., 2011). 
Diante do exposto, o presente estudo objetivou por descrever a taxa possíveis/prováveis transtornos de Ansiedade e Depressão, bem como sua possível concomitância, entre acadêmicos ingressados em um curso de Medicina na região Norte de Santa Catarina. Assim como os métodos escolhidos pelos mesmos para confrontar tais transtornos.

\section{MÉTODOS}

Foi realizado um estudo descritivo observacional prospectivo durante o segundo semestre de 2019 a partir de dados obtidos de estudantes de um curso de Medicina da Região Norte-nordeste de Santa Catarina, Brasil. Para participar do estudo os alunos deveriam estar matriculados, ter idade igual ou superior a 18 anos, assinar o Termo de Consentimento Livre e Esclarecido e responder integralmente o questionário aplicado.

Para avaliar a presença de sintomas de depressão e ansiedade foi utilizada a Escala HAD (Avaliação do nível de ansiedade e depressão). Essa escala possui 14 itens, sendo sete para ansiedade (questões $1,3,5,7,9,11,13)$ e sete para depressão $(2,4,6,8,10,12$ e 14$)$. A pontuação para cada item varia de zero a três, com três denotando o nível mais alto de ansiedade ou depressão e permite a seguinte categorização dos respondentes como Improvável (0 - 7 pontos), Possível (8 - 11 pontos) e Provável (12 - 21 pontos).

A grande vantagem apresentada pela HAD é a ausência de sintomas comuns entre depressão e doenças clínicas, como cansaço excessivo, anorexia e alterações no ciclo vigília-sono. A avaliação de sintomas subjetivos mais específicos também se torna possível com essa ferramenta, demonstrando com seus resultados a necessidade do uso de outros métodos adequados de diagnóstico, na identificação e na conduta terapêutica. Destacam que o instrumento tem boa sensibilidade $(70,8 \%$ a $80,6 \%)$ e especificidade $(69,6 \%$ a $90,9 \%$ ) em comparação a outras escalas como a Escala de Ansiedade de Beck (EAB) e à Escala de Depressão de Beck (EDB). Tais características justificaram a escolha dessa escala para compor 0 questionário (VASCONCELOS TC, et al., 2015).

Além da escala HAD, foram coletados dados sobre idade, gênero, semestre cursado, uso de substância para controle da ansiedade/depressão (nicotina, álcool, plantas medicinais, drogas ilícitas), realização de atividades físicas, acompanhamento psiquiátrico e/ou psicológico.

Para a coleta dos dados, utilizou-se um questionário de autopreenchimento que foi aplicado em apenas um encontro e sem necessidade de entrevista com os participantes. Para aplicação dos questionários foi solicitada autorização de professores do curso para a utilização de alguns minutos de suas aulas.

O projeto foi autorizado pelo Comitê de Ética em Pesquisa com Seres Humanos da UNIVILLE sob parecer ํo 2.801.781.

\section{RESULTADOS}

Durante o período estabelecido para a coleta dos dados 232 acadêmicos responderam ao questionário aplicado correspondendo a $60,4 \%$ dos alunos regularmente matriculados do primeiro ao oitavo semestre no curso de medicina da universidade. Com relação ao gênero, $71 \%(n=165)$ se declararam mulheres. Quando a idade foi avaliada, verificou-se variação dos 18 aos 35 anos, porém mais de $70 \%$ estavam na faixa dos 20 aos 25 anos.

A Figura 1 demonstra os resultados da análise dos dados obtidos pela escala HAD nas questões relacionadas a ansiedade e a depressão. 
Figura 1 - Prevalência de ansiedade depressão segunda a Escala HAD dentre os respondentes.

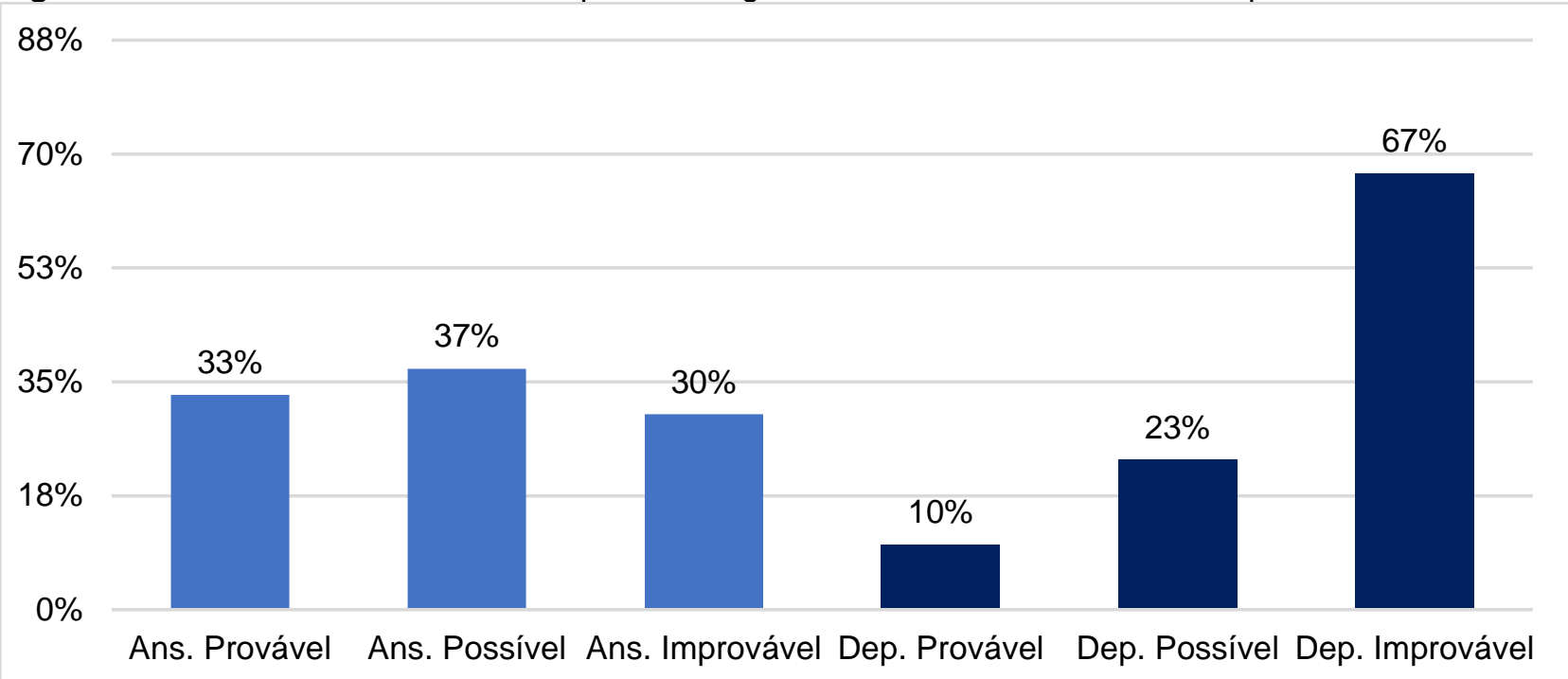

Fonte: Pinheiro EB, et al., 2021.

Quando questionados sobre o uso de alguma substância para controle da ansiedade e/ou depressão, 94 $(40,5 \%)$ afirmaram utilizar medicamentos ansiolíticos e/ou antidepressivos ( $n=60)$, álcool, nicotina, cannabis e outras substâncias como ecstasy, LSD e cocaína $(n=41)$. Observou-se que dentre os que utilizavam medicamentos, 47 (78\%) eram mulheres e 13 eram homens (22\%). Já no grupo que citou a utilização de álcool, nicotina, cannabis e outras substâncias como ecstasy, LSD e cocaína, 22 eram mulheres (53,6\%) e $19(46,3 \%)$ eram homens.

Foi realizada análise dos dados relacionados aos acadêmicos que obtiveram resultados de ansiedade e depressão provável (escore acima de 11 pontos). A análise de dados por semestre demonstrou maior prevalência de ansiedade e depressão provável entre os acadêmicos do sexto semestre do curso (Tabela 1).

Tabela 1 - Porcentagem de participantes com ansiedade ou depressão provável segundo escala HAD analisados a cada semestre do curso.

\begin{tabular}{c|c|c}
\hline Semestre (amostra) & Participantes com ansiedade provável & Participantes com depressão provável \\
\hline $1(n=31)$ & $9(29 \%)$ & $2(6,4 \%)$ \\
\hline $2(n=35)$ & $10(28,6 \%)$ & $1(2,8 \%)$ \\
\hline $3(n=11)$ & $4(36,4 \%)$ & $1(9 \%)$ \\
\hline $4(n=23)$ & $8(34,8 \%)$ & $3(13 \%)$ \\
\hline $5(n=36)$ & $14(38,9 \%)$ & $5(13,9 \%)$ \\
\hline $6(n=24)$ & $10(41,7 \%)$ & $5(21 \%)$ \\
\hline $7(n=39)$ & $15(38,5 \%)$ & $5(13 \%)$ \\
\hline $8(n=33)$ & $6(18,2 \%)$ & $1(3 \%)$ \\
\hline
\end{tabular}

Fonte: Pinheiro EB, et al., 2021.

Também foi possível identificar 19 participantes (8,2\%) que obtiveram pontuação compatível com quadro provável de depressão e ansiedade associadas. Esse grupo foi formado por cinco alunos do quinto semestre, quatro do sexto, quatro do sétimo, três do quarto e um do primeiro. Dessas 19 pessoas, 12 afirmaram realizar acompanhamento com psicólogo e/ou psiquiatra sendo que 11 iniciaram após o ingresso na faculdade. 
Quando foram analisados os dados por semestre do curso referentes a ansiedade e depressão possível, observou-se maior prevalência de ansiedade possível no terceiro e oitavos semestres e de depressão possível no sexto semestre (Tabela 2).

Tabela 2 - Porcentagem de participantes com ansiedade ou depressão possível segundo escala HAD analisados a cada semestre do curso.

\begin{tabular}{c|c|c}
\hline Semestre (amostra) & Participantes com ansiedade possível & Participantes com depressão possível \\
\hline $1(n=31)$ & $12(38,7)$ & $8(25,8 \%)$ \\
\hline $2(n=35)$ & $12(34,2 \%)$ & $6(17,1 \%)$ \\
\hline $3(n=11)$ & $5(45,45 \%)$ & $2(11 \%)$ \\
\hline $4(n=23)$ & $9(39,13 \%)$ & $7(30,4 \%)$ \\
\hline $5(n=36)$ & $13(36,11 \%)$ & $6(16,6 \%)$ \\
\hline $6(n=24)$ & $10(41,66)$ & $\mathbf{9 ( 3 7 , 5 \% )}$ \\
\hline $7(n=39)$ & $11(28,20 \%)$ & $7(21,2 \%)$ \\
\hline $8(n=33)$ & $\mathbf{1 5 ( 4 5 , 4 5 \% )}$ & $8 \%)$ \\
\hline
\end{tabular}

Fonte: Pinheiro EB, et al., 2021.

\section{Análise dos dados de ansiedade provável}

Como anteriormente mencionado, foram detectados 76 participantes com pontuação igual ou superior a 12 caracterizando ansiedade provável. Com relação ao gênero, $82 \%(n=62)$ eram mulheres. $51 \%$ dos entrevistados $(n=39)$ afirmou estar em acompanhamento com psicólogo e/ou psiquiatra sendo possível identificar dentre esses 39 casos, 26 (67\%) tiveram diagnóstico e iniciaram o acompanhamento após o ingresso na universidade.

Quarenta e quatro (58\%) dos 76 participantes com ansiedade provável afirmaram utilizar alguma substância para alívio da ansiedade destacando-se medicamentos ansiolíticos e/ou antidepressivos ( $n=31)$; álcool ( $n=16)$; cigarros, cigarros eletrônicos ou narguilés $(n=08)$; maconha $(n=06)$; fitoterápicos $(n=05)$; ecstasy, cocaína, LSD e outros ( $n=02)$.

Dentre os 31 que citaram medicamentos 15 utilizavam apenas um medicamento, 11 utilizavam dois medicamentos e cinco utilizavam três ou mais medicamentos chegando-se a um participante que afirmou usar seis medicamentos diferentes. Escitalopram $(n=10)$, desvenlafaxina $(n=09)$, e bupropiona $(n=05)$ foram os mais citados. Chamam a atenção quatro casos (13\%) em que o uso de medicamentos não era acompanhado por psiquiatra.

Com relação a realização de atividades para o controle da ansiedade, 51 (67\%) participantes afirmaram realizar frequentemente alguma atividade sendo a musculação a mais citada ( $n=37)$.

\section{Análise dos dados de depressão provável}

Ao todo 23 participantes (9,9\%) obtiveram pontuação igual ou maior que 12 na escala HAD, sendo classificados assim como depressão provável. Neste cenário, o número de participantes mulheres que obtiveram essa pontuação foi de 21 , representando cerca de $91,3 \%$, contra apenas dois participantes homens. Nesse grupo de 23 alunos, 15 realizavam acompanhamento com psicólogo ou psiquiatra e desses, 14 iniciaram após ingresso na universidade. Dezessete entrevistados (74\%) apontaram o uso de substâncias para controle da depressão com destaque para os medicamentos ansiolíticos e/ou antidepressivos $(n=14)$ em especial bupropiona, venlafaxina e desvenlafaxina. Houve um mesmo número de participantes que afirmou 
utilizar um medicamento( $n=06)$ e dois medicamentos $(n=06)$. Em dois casos $(14,3 \%)$ ainda que houvesse uso de medicamentos o entrevistado não realizava acompanhamento com psicólogo ou psiquiatra.

Diferentemente do que foi visto no grupo com ansiedade provável, aqui $56 \%$ dos entrevistados $(n=13)$ não realiza atividades físicas como forma de alívio da depressão.

\section{Análise dos dados de ansiedade possível}

Dos entrevistados, 87 obtiveram resultados dentro dessa margem, o que equivale à $37,50 \%$ do número total de participantes. Dentre os participantes, uma total de 67 são mulheres e 20 são homens, representando $41 \%$ e $30 \%$ de seus grupos, respectivamente. $72 \%$ dos participantes ( $n=63$ ) informou não realizar acompanhamento psicológico ou psiquiátrico. Dentre os que afirmaram realizar acompanhamento $(n=24), 19$ iniciaram após o ingresso na universidade. Para controle da ansiedade 49\% $(n=43)$ dos participantes classificados com ansiedade possível, realizam alguma atividade física sendo a musculação a mais frequente.

A maioria dos entrevistados ( $n=56$ ) afirmou não utilizar substâncias para controle da ansiedade. Dentre os que utilizam alguma substância, os medicamentos foram os mais citados $(n=22)$ com destaque para 0 escitalopram e a desvenlafaxina havendo um predomínio de monoterapia $(n=14)$. Seis dos 22 participantes que utilizavam medicamentos $(27,3 \%)$ informou não realizar acompanhamento com psicólogo ou psiquiatra.

\section{Análise dos dados de depressão possível}

Foram detectados o total de $53(22,8 \%)$ participantes que se enquadram no patamar de possível depressão sendo desses 44 (83\%) mulheres. Dezenove participantes (36\%) estavam em acompanhamento com psicólogo e/ou psiquiatra sendo que desses, apenas quatro iniciaram antes do ingresso na universidade.

Trinta e um (58,5\%) entrevistados disseram não utilizarem substância para controle da depressão. Dentre os que utilizam ( $n=22), 15(68,2 \%)$ utilizam medicamentos, cinco em monoterapia e cinco em terapia combinada com dois antidepressivos e/ou ansiolíticos destacando-se desvenlafaxina e bupropiona. Também foram citados álcool $(n=07)$, nicotina $(n=04)$, cannabis $(n=02)$. Novamente a maioria dos participantes $(n=33)$ afirmou realizar atividades físicas como forma de controle da depressão sendo a musculação a mais citada $(n=28)$.

\section{DISCUSSÃO}

Dentre as desordens psiquiátricas, a depressão e a ansiedade são as mais comuns sendo frequentemente pesquisadas dentre acadêmicos que se preparam para a carreira médica (LEÃO AM, et al., 2018). No presente estudo aproximadamente $33 \%$ e $10 \%$ dos participantes apresentaram sintomas compatíveis com quadro de ansiedade e depressão provável sendo a prevalência maior em mulheres. Uma revisão integrativa publicada em 2021 avaliou a prevalência de depressão e ansiedade entre estudantes de medicina brasileiros e encontrou taxas variando de $5,6 \%$ a $45,7 \%$ para depressão e de $13,4 \%$ a $41,4 \%$ para ansiedade (MENDES TC e DIAS ACP, 2021). Com relação ao gênero outros estudos similares realizados em território nacional também demostraram maior frequência de transtornos de ansiedade e depressão em mulheres (LEÃO AM, et al., 2018; MAYER FB, 2017; COSTA DS, et al., 2020).

Os estudos apontam diferenças na prevalência de depressão e ansiedade de acordo com o ano ou semestre cursado pelos alunos. Há estudos demonstrando maior prevalência nos primeiros anos do curso e outros apontando maior frequência ao final do curso (BASSOLS AM, et al., 2017; COSTA DS, et al., 2020; PEREIRA GA, et al., 2015; CAMPOS JCL, et al., 2017). No presente estudo encontrou-se maior prevalência no sexto semestre correspondendo a metade do curso. Acredita-se que esse fator esteja relacionado a grande carga horária observada neste semestre, 792 horas-aula, em comparação aos demais do curso avaliado.

Outro ponto que chamou a atenção foi o fato de a maioria dos participantes que obtiveram pontuação compatível com quadros prováveis e possíveis de depressão e ansiedade e que afirmaram realizar acompanhamento com psicólogo e/ou psiquiatra, diz ter iniciado os atendimentos após o ingresso na universidade. 
Diversos fatores como as características pessoais de cada acadêmico e a maneira como enfrentam o estresse, parecem estar associados ao desenvolvimento de transtornos de ansiedade e depressão, mas a forma como ocorrem os treinamentos ao longo do curso também parece influenciar o aparecimento de transtornos mentais (RIBEIRO CF, et al., 2020).

Longas cargas horárias de trabalho mental e uma redução na qualidade do sono e bem-estar geral são fatores associados ao desenvolvimento de transtornos de ansiedade, depressão e abuso de substâncias (JANSEN EC, et al., 2021; CAZOLARI PG, et al., 2020; LEÃO AM, et al., 2018).

Sobre o acompanhamento psiquiátrico e/ou psicológico, estudos demonstram taxas em torno de $27 \%$ para casos de ansiedade e $13 \%$ a $24 \%$ para depressão (VASCONCELOS TC, et al., 2015). Esses valores são inferiores aos encontrados no presente estudo dentre aqueles com escore compatível com quadros de ansiedade e depressão provável que foi de $51 \%$ e $65 \%$ respectivamente.

A análise de dados referentes ao consumo de substâncias para controle da ansiedade e da depressão demonstrou predomínio de medicamentos psicotrópicos com destaque para bupropiona, venlafaxina, desvenlafaxina e escitalopram. Alguns estudos brasileiros que analisaram o consumo de medicamentos antidepressivos entre estudantes de medicina, apontam maior consumo de fluoxetina, escitalopram (RIBEIRO CF, et al., 2021; SOUZA RCS, et al., 2021; VELTER FILHO ML, 2020).

A relação de comorbidade entre o uso de álcool e outras drogas com transtornos de Humor é bem documentada. Uma revisão sistemática realizada pelo Departamento de Psiquiatria e Ciências de Saúde Comunitária, Universidade de Manitoba no Canadá concluiu que $24-27 \%$ da população analisada fazia uso de álcool ou drogas ilícitas com o intuito de aliviar sintomas ansiosos e/ou depressivos (TURNER S. et al., 2018).

Um estudo realizado em uma faculdade de medicina do espirito santo analisou o consumo de substancias psicoativas por estudantes de medicina, reportou que 70,3\% dos participantes consumiam álcool sendo que cerca de $32,2 \%$ era classificado como uso abusivo de álcool. Além disso 83,6\% faziam uso de drogas ilícitas esporadicamente (MIRANDA CC, et al., 2020).

Constatou-se também um aumento no consumo de álcool e substâncias ilícitas no decorrer do curso. Esse aumento poderia ser explicado pelo pouco tempo para socialização disponível e excesso de conteúdo, densa carga horária com o avançar do currículo (RODRIGUES IS, et al., 2021; RODRIGUES ALM, et al., 2020).

Outras pesquisas relacionam os benefícios psicológicos do exercício devido a fatores propriamente psicológicos e sociais como aumento da autoconfiança, humor, positividade, sensação de prazer, autoestima e relações interpessoais e fatores fisiológicos, como aumento do nível de serotonina e da aptidão física (KIM Y, et al., 2018; SILVA LC e SANTOS NML, 2019).

Além de minimizar os sintomas da depressão, o exercício físico melhora a função cognitiva e a qualidade do sono, sendo por isso instituída em guidelines de países como Suécia e Canadá para tratamento da depressão (GOURGOUVELIS J, et al., 2018; CARNEIRO LF, et al., 2017).

Neste cenário, é ressaltada a necessidade de implementação da atividade física como estratégia para a promoção de saúde mental e física nos acadêmicos do curso de Medicina, assim como a importância de orientação estudantil e treinamento para o manejo de tempo, permitindo a combinação entre atividades acadêmicas e estilo de vida saudável (MAIA HAA, et al., 2020; BUHRER BE, et al., 2019).

Neste estudo, evidenciou-se que mais de $60 \%$ daqueles com pontuação compatível com quadro de ansiedade provável e depressão possível, e mais de $40 \%$ dos que apresentaram escore indicando depressão provável e ansiedade possível, realizavam atividades físicas como forma de alívio da ansiedade e/ou da depressão. Comparativamente, um estudo realizado com internos do sexto ano de Medicina de universidades públicas e privadas de Santa Catarina constatou que cerca de $50 \%$ dos alunos realizavam atividade física sistematizada, com frequência semanal. Além disso, apesar dos elevados níveis de estresse a que eram submetidos, apresentavam qualidade de vida positiva (MEYER C, et al., 2012). 


\section{CONCLUSÃO}

Evidencia-se que, no grupo estudado, a taxa de acadêmicos com provável diagnóstico de Transtorno Ansioso ou Depressivo é de 33\% e 10\%, respectivamente. Dentre os quais, a maioria afirmou realizar alguma forma de atividade para o controla de depressão e/ou ansiedade, sendo musculação a mais citada. Os fármacos mais citados foram desvenlafaxina, bupropiona e escitalopram. A substância recreativa mais referida foi o álcool, seguida da nicotina. Estes achados refletem e solidificam a bibliografia já disponível na área, mostrando o risco acentuado que estudantes de medicina apresentam no desenvolvimento de Transtornos de Humor.

\section{REFERÊNCIAS}

1. BASSOLS AM, et al. How do medical students defend themselves against anxiety?. Revista brasileira de psicoterapia, 2017; 19(1): 31-42.

2. BUHRER BE, et al. Análise da qualidade e estilo de vida entre acadêmicos de medicina de uma instituição do Norte do Paraná. Revista Brasileira de Educação Médica, 2019; 43(1): 39-46.

3. CAMPOS JCL, et al. Avaliação do nível de andiedade e depressão dos estudantes de medicina do UNIFESO. Revista da JOPIC, 2020; 3(7): 40-55.

4. CARNEIRO LF, et al. Portuguese and brazilian guidelines for the treatment of depression: Exercise as medicine. Revista Brasileira de Psiquiatria, 2017; 40(2): 210-1.

5. CAZOLARI PG, et al. Níveis de burnout e bem-estar de estudantes de medicina: um estudo. Revista Brasileira de Educação Médica, 2020; 44(4): 8.

6. COSTA DS, et al. Sintomas de depressão, ansiedade e estresse em estudantes de medicina e estratégias institucionais de enfrentamento. Revista Brasileira de Educação Médica, 2020; 44(1): e040.

7. GOURGOUVELIS J, et al. Exercise leads to better clinical outcomes in those receiving medication plus cognitive behavioral therapy for major depressive disorder. Front Psychiatry, 2018; 9(1): 1-12.

8. JANSEN EC, et al. Associations between mental workload and sleep quality in a sample of young adults recruited from a US college town. Behavioral Sleep Medicine, 2020; 18(4): 513-522.

9. KIM Y, Effect of university students' sedentary behavior on stress, anxiety, and depression. Perspect Psychiatric Care, 2019; 55(1): 164-169.

10. LEÃO AM, et al. Prevalência e fatores associados à depressão e ansiedade entre estudantes universitários de área da saúde em um grande centro urbano do Nordeste do Brasil. Revista Brasileira de Educação Médica, 2018; 42(4): $55-65$.

11. MACHADO SLM, et al. Ansiedade e depressão em estudantes de medicina. Revista Saúde Multidisciplinar, 2019; 6(2).

12. MAIA HAA, et al. Prevalência de Sintomas Depressivos em Estudantes de Medicina com Currículo de Aprendizagem Baseada em Problemas. Revista brasileira de educação médica. 2020; 44(3): e105.

13. MAYER FB. A prevalência de sintomas de depressão e ansiedade entre os estudantes de medicina: um estudo multicêntrico no Brasil. (Tese em Ciências) - Programa de Ciências Médicas. Universidade de São Paulo, São Paulo, 2017; $119 \mathrm{p}$.

14. MEC. Ministério da Educação. Notas de corte. 2020.

15. MENDES TC, DIAS ACP. Symptoms of depression, anxiety, stress and associated factors in brazilian medicina studentes: an integrative review. Research, Society and Development, 2021; 10(4): e14910414033.

16. MEYER C, et al. Qualidade de vida e estresse ocupacional em estudantes de medicina. Revista Brasileira de Educação Médica, 2012; 36(4): 489-498.

17. MIRANDA CC, et al. Análise do consumo de substâncias psicoativas por estudantes de medicina de uma faculdade do Espirito Santo, Brasil. Arquivos Médicos dos Hospitais e da Faculdade de Ciências Médicas da Santa Casa de São Paulo, 2020; 65: e33.

18. OWENS H, et al. Sleep behaviors in traditional-age college students: A state of the science review with implications for practice. Journal of the American Association of Nurse Practitioners, 2017; 29(11): 695-703.

19. PAULA JA, et al. Prevalência e fatores associados à depressão em estudantes de medicina. Revista Brasileira do Crescimento e Desenvolvimento Humano, 2014; 24(3): 274-281.

20. PEREIRA GA, et al. Prevalência de síndromes funcionais em estudantes e residentes de medicina. Revista Brasileira de Educação Médica, 2015; 39(3): 395-400.

21. QUICK V, et al. Eat, sleep, work, play: Associations of weight status and health-related behaviors among young adult college students. American Journal of Health Promotion, 2014; 29(2): e54-e72. 
22. RIBEIRO AG, et al. Antidepressivos: Uso, adesão e conhecimento entre estudantes de medicina. Ciência \& Saúde Coletiva, 2014; 19(6): 1825-1833.

23. RIBEIRO CF, et al. Prevalência de fatores associados à depressão e ansiedade em estudantes de medicina brasileiros. Revista Brasileira de Educação Médica, 2020; 44(1): e021

24. RIBEIRO AGS, et al, Transcamentos de matrículo no curso de medicina da UFMG: Sintomas de sofrimento psíquico. Revista Brasileira de Educação Médica, 2016; 40(4): 583-590.

25. ROBERTO A, et al. A saúde mental de estudantes de medicina estudo exploratório na universidade da Beira interior. Acta Médica Portuguesa, 2011; 24 (suppl.2): p. 279-286.

26. RODRIGUES ALM, et al. Udo de álcool, tabaco e outras substâncias psicoativas entre discentes do curdo de medicina: um estudo transverso. Pará Research Medical Journal, 2020; 4: e40.

27. RODRIGUES IS, et al. Consumo de álcool por acadêmicos de medicina de uma Universidade Pública no Estado do Pará. Research, Society and Development, 2021; 10(3): e58310313404.

28. SILVA LC, SANTOS NML. Efeitos do exercício físico nos aspectos fisiológicos, psicológicos e sociais em pessoas com depressão. Revista Científico Eletrônica de Ciências Aplicas da FAIT, 2019; 14(2).

29. SOUZA RCS, et al. O uso de antidepressivos em estudantes da área da saúde. Brazilian Journal of Development, $2021 ; 7(4): 40842-40852$.

30. TURNER S, et al. Self-medication with alcohol or drugs for mood and anxiety disorders: A narrative review of the epidemiological literature. Depression and Anxiety, 2018; 35(9): 851-860.

31. VASCONCELOS TC, et al. Prevalência de Sintomas de Ansiedade e Depressão em Estudantes de Medicina. Revista Brasileira de Educação Médica, 2015; 39(1): 135-142.

32. VELTER FILHO ML, et al. Análise do uso de antidepressivos e psicoestimulantes e seus efeitos sobre acadêmicos de medicina de uma universidade da região noroeste do Paraná, 1ª Ed. Ponta Grossa: ATENA, 2020. 